\title{
3dSpAn: An interactive software for 3D segmentation and analysis of dendritic spines
}

\author{
Nirmal Das ${ }^{1, \ddagger}$, Ewa Baczynska ${ }^{2,3} \ddagger$, Monika Bijata ${ }^{2}$, Blazej Ruszczycki ${ }^{8}$, Andre Zeug ${ }^{7}$, Dariusz \\ Plewczynski $^{4,5}$, Punam Kumar Saha ${ }^{6}$, Evgeni Ponimaskin ${ }^{7}$, Jakub Wlodarczyk ${ }^{2, *}$ and Subhadip \\ Basu $^{1, *}$
}

1 Department of CSE, Jadavpur University, 188 Raja S.C. Mullick Road, Kolkata-700032, India; subhadip.basu@jadavpuruniversity.in (S.B.); nirmaldas.cse.rs@jadavpuruniversity.in (N.D.)

2 Laboratory of Cell Biophysics, The Nencki Institute of Experimental Biology, Polish Academy of Sciences, Pasteura 3, 02-093, Warsaw, Poland; e.baczynska@nencki.edu.pl (E.B.); m.bijata@nencki.edu.pl (M.B.); j.wlodarczyk@nencki.edu.pl (J.W.)

3 The Institute of Physical Chemistry, Polish Academy of Sciences, Kasprzak Street 44/52, 01-224, Warsaw, Poland. (E.B.)

4 Laboratory of Bioinformatics and Computational Genomics, Faculty of Mathematics and Information Science, Warsaw University of Technology, Warsaw, Poland; d.plewczynski@cent.uw.edu.pl (D.P.)

5 Laboratory of Functional and Structural Genomics, Centre of New Technologies, University of Warsaw, Warsaw, Poland. (D.P.)

6 Department of Electrical and Computer Engineering \& Department of Radiology, University of Iowa, Iowa City, IA 52242, USA; pksaha@healthcare.uiowa.edu (P.K.S.)

7 Cellular Neurophysiology, Hannover Medical School, 1 Carl-Neuberg Street, 30625, Hannover, Germany; zeug.andre@mh-hannover.de (A.Z.); ponimaskin.evgeni@mh-hannover.de (E.P.)

8 Laboratory of Imaging Tissue Structure and Function, The Nencki Institute of Experimental Biology, Polish Academy of Sciences, Pasteura 3, 02-093, Warsaw, Poland; b.ruszczycki@nencki.edu.pl (B.R.)

* Correspondence: subhadip.basu@jadavpuruniversity.in (S.B.), j.wlodarczyk@nencki.gov.pl (J.W.)

$\ddagger$ These authors contributed equally to this work.

Version December 27, 2020 submitted to Journal Not Specified

\begin{abstract}
Three dimensional segmentation and analysis of dendritic spines involve two major challenges: 1) how to segment individual spines from the dendrites and 2) how to quantitatively assess the morphology of individual spines. We developed a software named 3dSpAn to address these two issues by implementing our previously published 3D multiscale opening algorithm in shared intensity space and using effective morphological features for individual dendritic spine plasticity analysis. 3dSpAn consists of four modules: Preprocessing and ROI selection, Intensity thresholding and seed selection, Multiscale segmentation and Quantitative morphological feature extraction. We show the results of segmentation and morphological analysis for different observation methods, including in vitro and ex vivo imaging with confocal microscopy, and in vivo samples, using high-resolution two-photon microscopy. The software is freely available, the source code, windows installer, the software manual and video tutorial can be obtained from: https://sites.google.com/view/3dSpAn/.
\end{abstract}

Keywords: Dendritic spine; 3D segmentation; Interactive software; Quantitative analysis.

\section{Introduction}

In this article, we introduce an interactive software, 3dSpAn, designed to segment and quantitatively assess 3D morphology of dendritic spines. We evaluate the software performance with different imaging conditions (in vitro, ex vivo and in vivo). The software is based on an algorithm presented in our previous work [1], which provides a detailed theoretical background and validation 
of the proposed method with respect to the available state-of-the-art tools, such as Imaris software [2], demonstrating a high multi-user reproducibility. The developed software uses 3D multiscale opening (MSO) algorithm [3] to segment the spines from the dendritic segments.

The present paper is an application note, focusing on the software usage, and aiming to reduce user interaction in the segmentation process, and to help with estimation of adjustable segmentation parameters, with enhanced visualization. The software is freely available under GNU Lesser General Public License version 3 (LGPL V3). The software executable (3dSpAn V1.2 Installer for Windows), the source code, the detail user guide and video tutorials are available at: https://sites.google. $\mathrm{com} /$ view/3dSpAn/. A comprehensive user manual is also given as a supplementary document 3dSpAn_Supplementary (also available as Manual in: https://sites.google.com/view/3dSpAn/). The complete workflow of the 3dSpAn software is described by the Figure S1-Figure S33 and Table S1 in the 3dSpAn_Supplementary. Additionally, Video S1, Viedo S2 and Video S3 will describe how to perform segmentation and analysis of individual dendritic spine in 3dSpAn and how to visualize the segmented spines in 3D.

Dendritic spines are small membranous protrusions on neuronal dendrites having distinct structural features [4], controlling electrical and biochemical compartmentalization and playing major roles in activity and signal transmission of neural circuits [5]. The shape of dendritic spines changes spontaneously, or in response to neuronal stimulation [6,7]. These changes are related with learning and memory [8] and many neuropsychiatric and neurodegenerative diseases $[9,10]$ e.g. Alzheimer's disease [11], schizophrenia [12]. Many aspects of the existing structure-function relationship in dendritic spines are still unknown due to their complex morphology $[8,10]$.

It is still challenging to segment individual spine and find exact spine boundaries, especially for lower-resolution microscopic images (when the possible highest resolution cannot be achieved, e.g. in in vivo imaging), that create difficulties in accurate modeling of 3D morphology of individual spine. The existing methods of segmentation and morphological analysis of dendritic spines either use 2D maximum intensity projection (MIP) image obtained from microscopic 3D image, or directly using microscopic 3D image. For example, the method presented in [13-15,21] use 2D MIP images for quantitative assessment of morphological changes in dendritic spines. However, 2D MIP images are misleading due to the loss of information and structure overlapping. Therefore, accurate quantitative morphological analysis of individual dendritic spine from 2-D MIP images is nearly impossible. Hence, the presented software segments individual dendritic spine directly in 3D and performs a morphological analysis of segmented dendritic spines. Several studies in the literature addressed the issue of segmentation and morphological analysis of dendritic spines directly for 3D microscopic images. A commercially available tool Imaris [2] allows user for 4D analysis of dendritic spine. Imaris is good for analyzing overall spine population but it fails to assess individual spine morphometry. In [17], Swanger et al proposed a method for automatic 4D analysis of dendritc spine morphology which follows the same pipeline as Imaris, however it does not quantify individual spine morphology. Several other methods are based on conventional machine learning as well as deep learning for segmentation and analysis of dendritic spine from microscopic images have been reported in the literature [32-34] but none of them is truely a 3D method. The main disadvantage of using deep learning based methods for segmentation and analysis of dendritic spines in 3D is the absence of sufficient manually annotated data in 3D. The works in $[35,36]$ used deep learning for reconstruction of synapses from 3D microscopic images and identification of 3D morphological motifs but these are not suitable for segmentation and analysis of individual dendritic spine in 3D. Thus, a software allowing for interactive segmentation and morphological analysis of individual dendritic spine in 3D is still missing.

\section{Results and Discussion}

We show the results of analysis performed with images originating from three different laboratory techniques, in vitro, ex vivo, and in vivo. The details of these techniques and imaging modalities are described in Table 1. The morphological feature values (volume, length, head width and neck length) 
bioRxiv preprint doi: https://doi.org/10.1101/864587; this version posted December 27, 2020. The copyright holder for this preprint (which was not certified by peer review) is the author/funder. All rights reserved. No reuse allowed without permission.

of the segmented spines from confocal microscopic image of in vitro neuronal culture (refer Figure 4) are shown in Table 2. Figure 5 and Figure 6 show the segmentation results in ex vivo sample and in vivo sample respectively. Table 3 and Table 4 show the morphological feature values of the segmented spines in Figure 5 and Figure 6. Figure 7 shows different segmented regions in a single 3D image. We observed that the performance of the software, in segmentation and analysis of dendritic spines, remained unchanged regardless of the laboratory technique used for imaging, and imaging modalities.

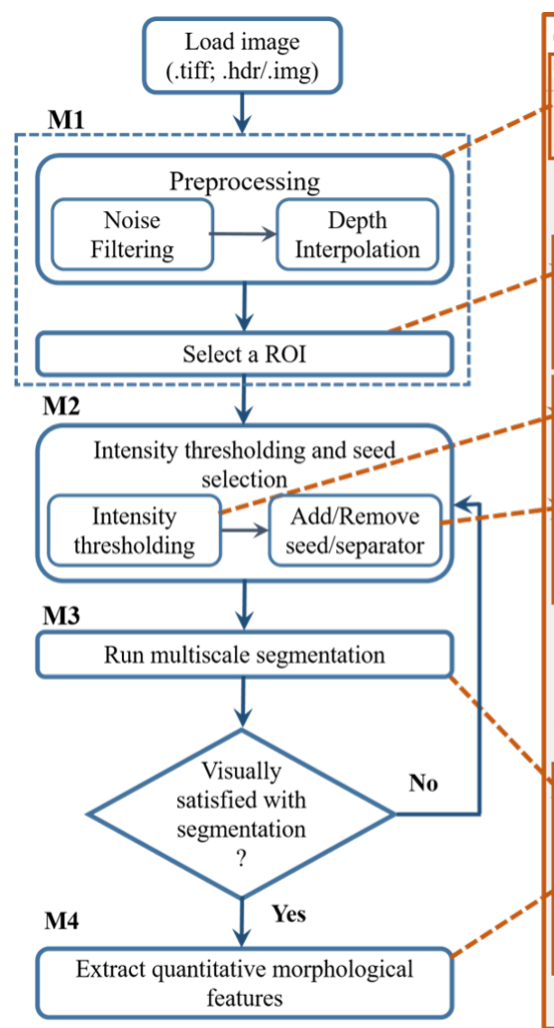

(a)

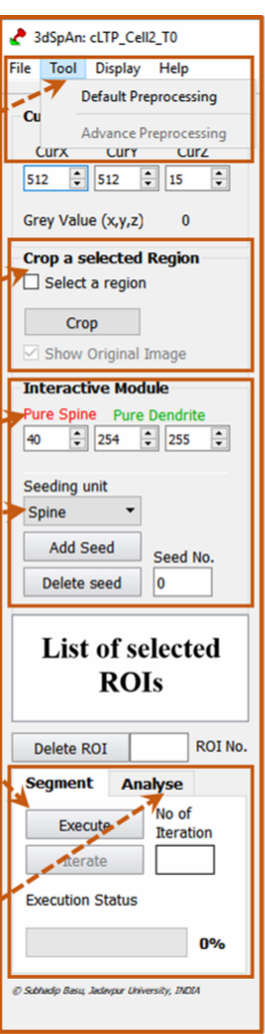

(b)

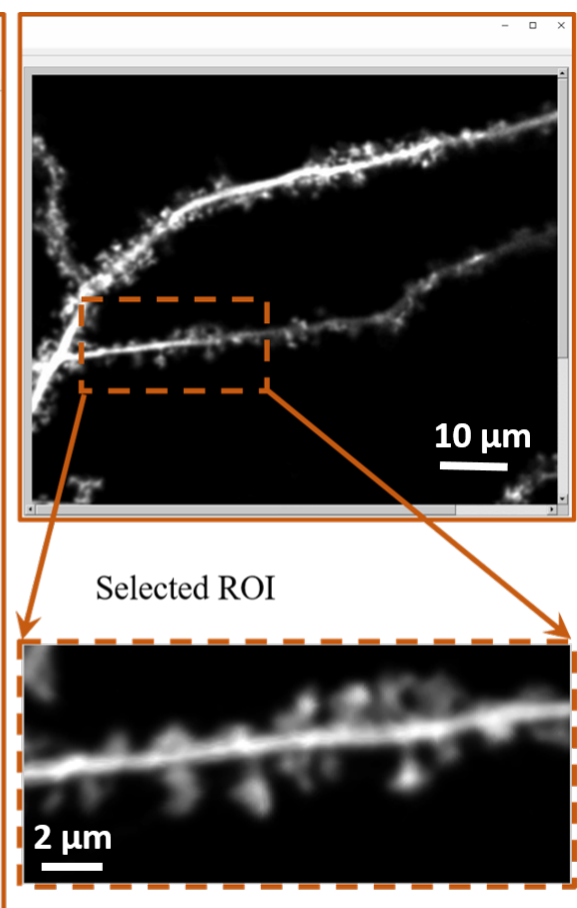

M1: Preprocessing and ROI selection

(c)

Figure 1. 3dSpAn workflow and brief description of the GUI and the M1 module. (a) Diagram of 3dSpAn workflow. The blocks are representing four main modules: M1, M2, M3 and M4 (b) GUI working panel. The main modules are enclosed with rectangular boxes, dashed arrows show the correspondence with the appropriate blocks of the workflow diagram. (c) An image of in vitro neuronal culture is loaded in GUI (above) and a ROI is selected (dotted rectangular box). The magnified version of selected ROI (Scale bar $=2 \mu \mathrm{m}$ ) is shown below the full image. 

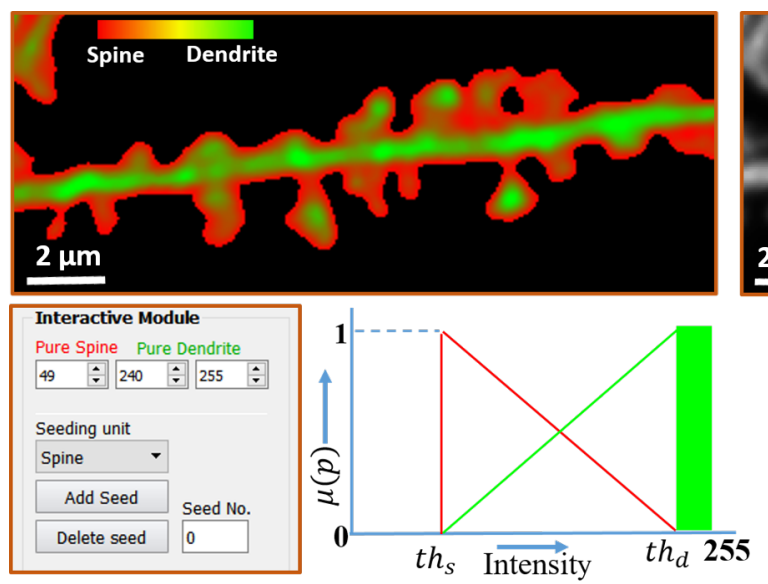

(a)

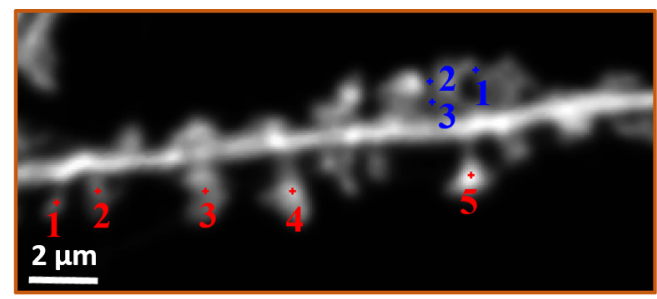

- Intensity thresholds are adjusted to include spines with higher intensity.

- Pixels with intensities greater than $t h_{d}$ are used as dendrite seeds.

(b)

M2: Intensity thresholding and seed selection

Figure 2. M2 module. (a) Visualization of the shared intensity space between spine and dendrite in the selected ROI (Scale bar $=2 \mu \mathrm{m}$ ) of Figure 1(c) with a color transition from red to green, describing the spine association and the dendrite association for the pixel intensity value (top). The block interactive module shows the intensity thresholds $t h_{s}(40)$ and $t h_{d}(240)$, for the spine and the dendrite respectively. The graph shows fuzzy membership curve for both spines (red) and the dendrite (green). (b) User-specified spine seeds (red) and separators (blue), placed at the same depth on different spines (Scale bar $=2 \mu \mathrm{m}$ ). The seeds are numbered in the order in which they were placed. Often, to include a spine, the user needs to adjust $t h_{s}$ and $t h_{d}$ values. The pixels with intensity value greater than $t h_{d}$ are used as dendrite seeds implicitly. For this ROI, explicit dendrite seeds are not given.
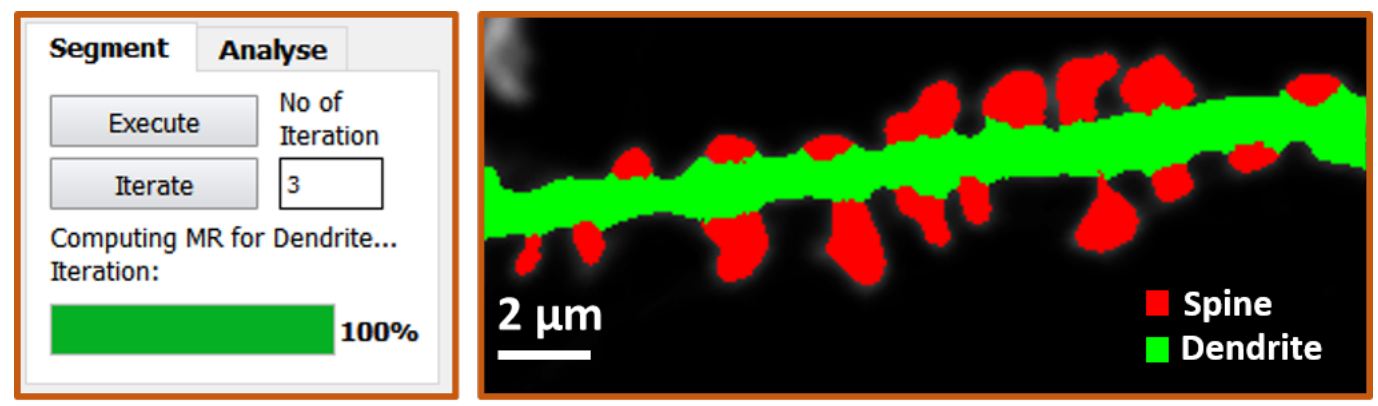

\section{M3: Segmentation Module}

Figure 3. M3 module and segmented spines (Scale bar=2 $\mu \mathrm{m}$ ) from the selected ROI of Figure 1(c). The segmentation module of the GUI shows the number of times the MSO algorithm iterates to achieve the segmentation result. 


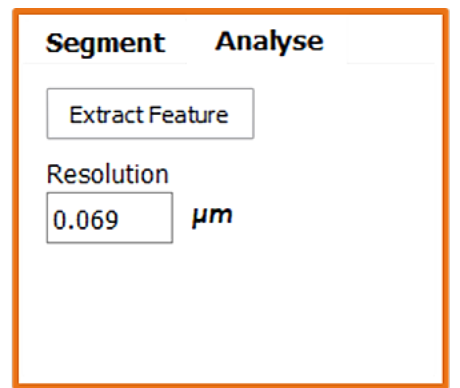

Spine no. 5:

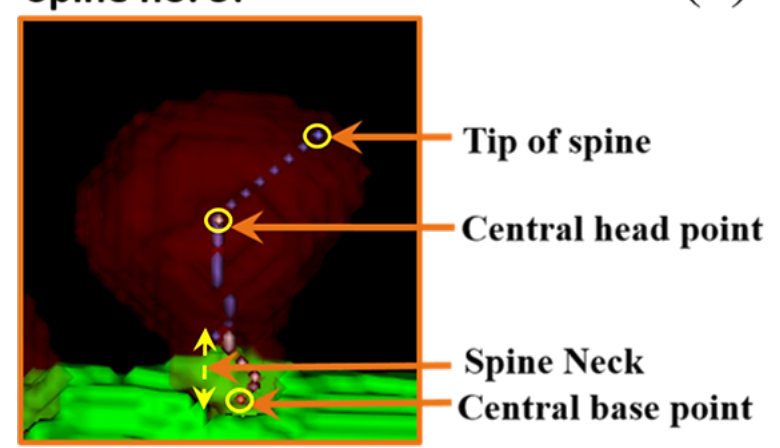

(b)

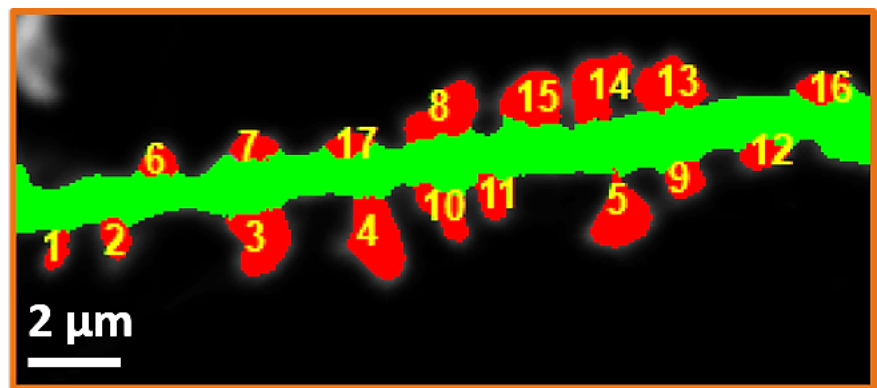

(a)

Vol: 1.418173 micron $^{3}$

Len: 1.311 micron

Head Width: 1.1454 micron

Neck length: 0.207 micron

Type: Mushroom

\section{M4: Quantitative feature extraction}

Figure 4. M4 module. (a) After quantitative morphological feature extraction, the segmented spines (Scale bar $=2 \mu \mathrm{m}$ ) (see Figure 3) are numbered in the same order as user-specified seed points. (b) Magnified and rotated 3D view of a spine no. 5. The spine is visualized with popular ITK-Snap software [19]. The transparent visualization helps to see the calculated path and points inside spine. The key points like Central base point, Central head point and Tip of the spine are marked (small yellow circle). Spine volume, spine length, head width and neck length are also calculated. Based on these measurements, spine classification is performed. Here the segmented spine belongs to the Mushroom class.
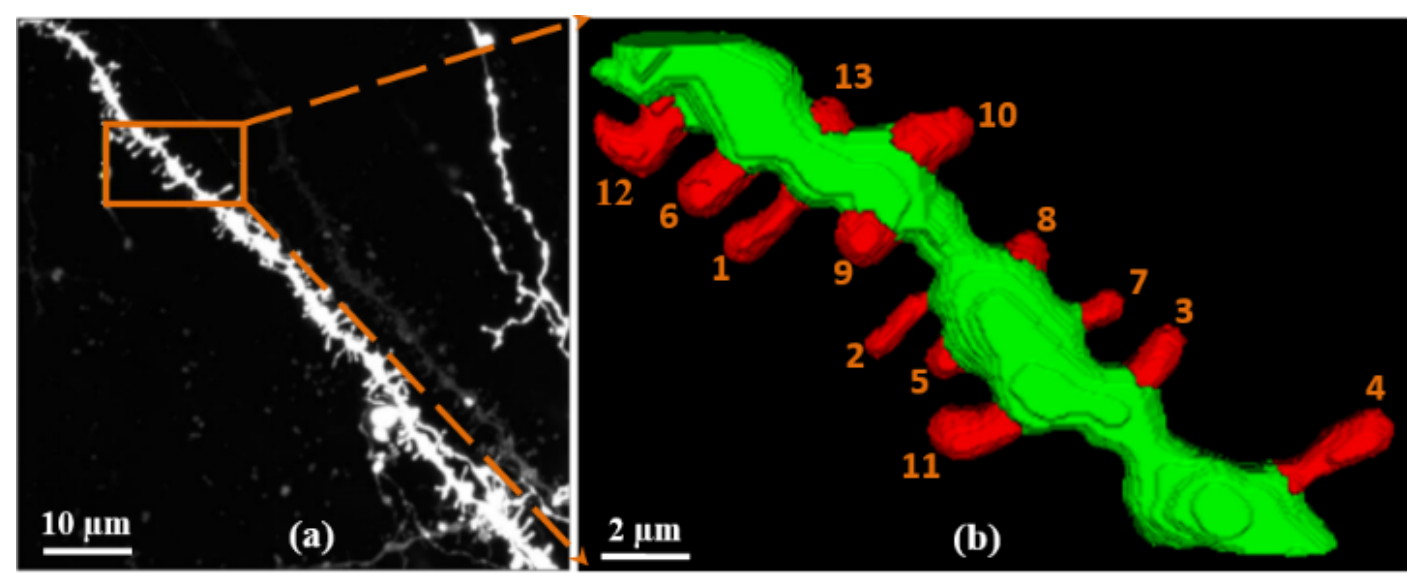

Figure 5. 3D reconstruction of the segmented spines (ex vivo). (a) A ROI is selected (shown in rectangular box) in an ex vivo image (Scale bar=10 $\mu \mathrm{m}$ ) of mouse brain slices. (b) Magnification of 3D reconstruction of the segmented spine and dendrite with individual spine numbering in the selected ROI(Scale bar $=2 \mu \mathrm{m})$. 

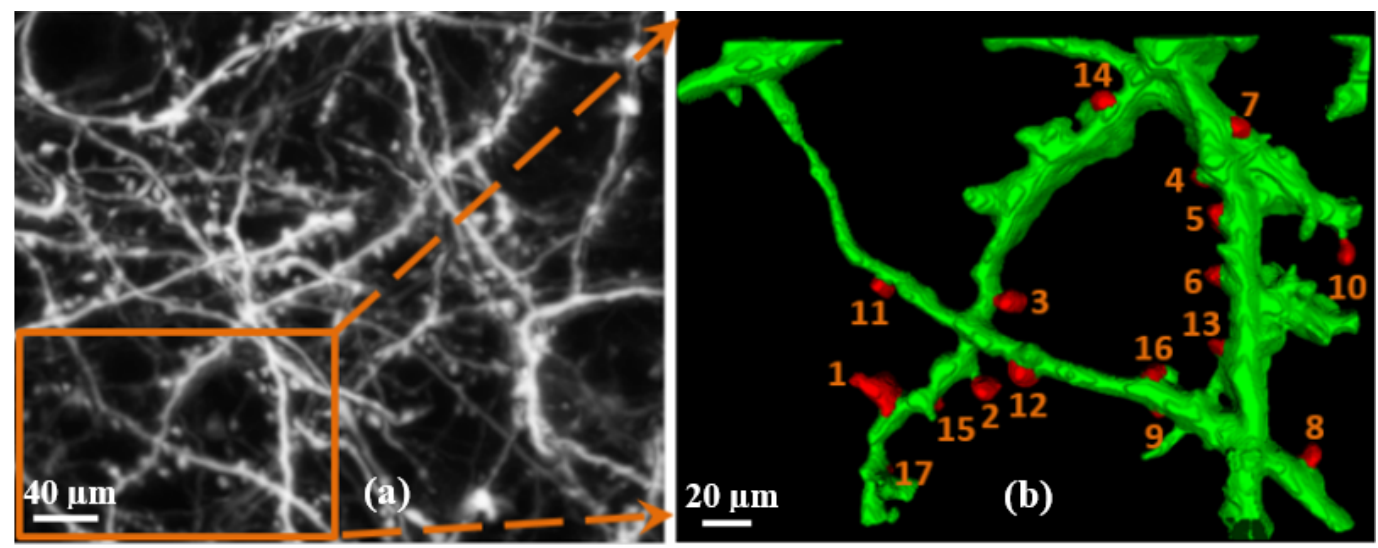

Figure 6. 3D reconstruction of the segmented spines (in vivo). (a) A ROI is selected in an in vivo image (Scale bar $=40 \mu \mathrm{m}$ ) of living mouse brain. (b) Magnification of 3D reconstruction of the segmented spine and dendrite with individual spine numbering in the selected ROI (Scale bar=20 $\mu \mathrm{m}$ ).

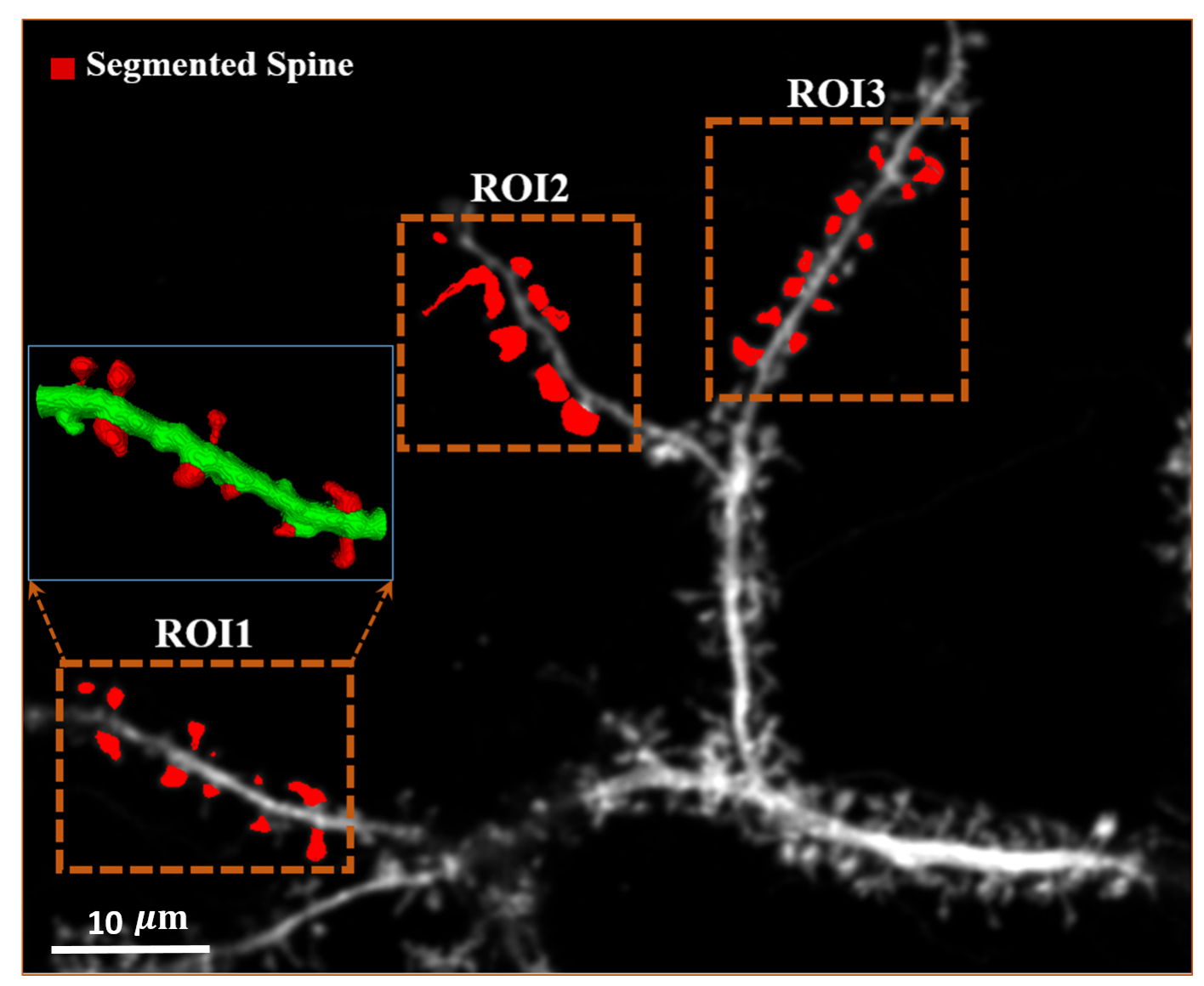

Figure 7. Different regions (ROI1, ROI2 and ROI3 are enclosed in dotted rectangular box) with segmented spines, confocal microscopic image of in vitro neuronal culture (Scale bar $=10 \mu \mathrm{m}$ ). 3D rendering of the ROI 1 is shown in the inset. Segmented spines are shown in red color. 
Table 1. Details of different imaging protocols.

\begin{tabular}{|c|c|c|c|}
\hline Methods & In-vitro & Ex-vivo & In-vivo \\
\hline Model & primary/dissociated neuronal culture & brain slices & living animal \\
\hline Animal & rat & mouse & mouse \\
\hline \multirow[t]{2}{*}{$\begin{array}{l}\text { Dendritic } \\
\text { spine } \\
\text { visualization } \\
\text { method }\end{array}$} & $\begin{array}{l}\text { transfection with EGFP plasmid under the control } \\
\text { of synapsin- } 1 \text { promoter at } 14 \text { day in vitro (DIV) of } \\
\text { hippocampal neurons }\end{array}$ & DiI staining on fixed tissue & $\begin{array}{l}\text { 2-photon imaging } \\
\text { by cranial } \\
\text { windows of } \\
\text { transgenic mouse } \\
\text { line (Thy1-GFP) } \\
\text { expressing GFP } \\
\text { under the thy1 } \\
\text { promoter allowing } \\
\text { visualization of } \\
\text { the neurons in } \\
\text { prefrontal cortex }\end{array}$ \\
\hline & GFP fluorescence & $\begin{array}{l}\text { DiI fluorescence } \\
\text { fluorescence channel) }\end{array}$ & $\begin{array}{l}\text { GFP/YFP } \\
\text { fluorescence }\end{array}$ \\
\hline $\begin{array}{l}\text { Protocols } \\
\text { used in: } \\
{[1,16]}\end{array}$ & $\begin{array}{l}\text { Primary hippocampal cultures prepared from } \\
\text { post-natal Day 0 Wistar rats. Hippocampi were } \\
\text { dissected on ice in dissociation medium DM ( } 81.8 \mathrm{mM} \\
\mathrm{Na} 2 \mathrm{SO} 4,30 \mathrm{mM} \mathrm{K} 2 \mathrm{SO} 4,5.8 \mathrm{mM} \text { dissected on ice } \\
\mathrm{MgCl} 2,0.25 \mathrm{mM} \mathrm{CaCl}, 1 \mathrm{mM} \mathrm{HEPES} \text { pH } 7.4,20 \mathrm{mM} \\
\text { glucose; } 1 \mathrm{mM} \text { kynureic acid; } 0.001 \% \text { Phenol Red) } \\
\text { and incubated twice for } 15 \mathrm{~min} \text { at } 37^{\circ} \mathrm{C} \text { with } 100 \text { units } \\
\text { of papain solutionPapain activity was stopped by } \\
\text { applying a trypsin inhibitor dissolved in DM. The } \\
\text { hippocampi were washed three times in plating } \\
\text { medium [PM;Minimum Essential Media; } 10 \% \text { fetal } \\
\text { bovine serum and } 1 \% \text { penicilin-streptomycin] and } \\
\text { triturated to homogeneous suspension and centrifuged } \\
\text { for } 10 \mathrm{~min} 1000 x g \text { resulting in cell pellet suspended in } \\
\text { plating medium. Cells were plated at density } 120000 \\
\text { cells per } 18 m m \text { diameter coverslip coated with } 1 \mathrm{mg} / \mathrm{ml} \\
\text { poly-L-lysine (Sigma-Aldrich) and } 2.5 \mu g / \mathrm{ml} \text { laminin } \\
\text { (Roche). After } 1.5 \mathrm{~h} \text { plating medium was exchanged for } \\
\text { maintenance medium (MM; Neurobasal-A, } 2 \% \mathrm{~B}-27 \\
\text { supplement and } 1 \% \text { penicillin-streptomycin). The cells } \\
\text { were maintained at } 37^{\circ} \mathrm{C} \text { under a humidified } 5 \% \mathrm{CO} 2 \\
\text { atmosphere. }\end{array}$ & $\begin{array}{l}\text { Biolistic staining with } \\
\text { DiI dye was performed } \\
\text { on C57BL6J mouse } \\
\text { brain slices. The mice } \\
\text { were anesthetized and } \\
\text { transcardially perfused with } \\
1.5 \% \text { paraformaldehyde. } \\
\text { The brains were sliced for } \\
140 \mu m \text { thick and allowed for } \\
1.5 h \text { at room temperature. } \\
\text { Random dendrite labeling } \\
\text { was performed using } 1.6 m m \\
\text { tungsten particles (Bio-Rad) } \\
\text { coated with propelled } \\
\text { lipophilic fluorescent dye } \\
\text { (1, } 1^{\prime} \text {-dioctadecyl-3,3,3, } 3^{\prime} \\
\text {-tetramethylindocarbocyanine } \\
\text { perchlorate,Invitrogen) } \\
\text { delivered to the cells by } \\
\text { gene gun (Bio-Rad). }\end{array}$ & $\begin{array}{l}\text { Virus injections } \\
\text { Implant cranial } \\
\text { windows } \\
\text { and inject } \\
\text { HAPLN1-Scarlet + } \\
\text { Synaptophysin-BFP } \\
\text { AAVs in PFC } \\
\text { for Thy1-GFP } \\
\text { mice. Imaging } \\
\text { after 4 weeks } \\
\text { postinjection: } \\
\text { Anaesthetize } \\
\text { with Isoflurane to } \\
\text { perform baseline } \\
\text { images (3 to } 4 \\
\text { positions) and } \\
\text { 24hours after } \\
\text { performance of } \\
\text { single image for } \\
\text { all positions. }\end{array}$ \\
\hline $\begin{array}{l}\text { Imaging } \\
\text { protocols }\end{array}$ & $\begin{array}{l}\text { Live cell imaging was performed at } 20-22 \\
\text { DIV. Cultures were transfected at } 14 \text { DIV with } \\
\text { Syn-GFP plasmid under a synapsin- } 1 \text { promoter with } \\
\text { Lipofectamine reagent. For imaging the cultures } \\
\text { were placed into the acquisition chamber under } 37^{\circ} \mathrm{C} \\
\text { temperature and } 5 \% \text { CO2 conditions. The images of } \\
\text { dendritic segments were acquired using Zeiss LSM780 } \\
\text { confocal microscope with a PL Apo } 40 x / 1.2 \mathrm{NA} \\
\text { water immersion objective using } 488 \mathrm{~nm} \text { diode-pumped } \\
\text { solid-state laser at } 10 \% \text { transmission at a pixel count } \\
1024 \times 1024 \text {. Z-stacks were collected for each cell with } \\
\text { a } 0.4 \mu m \text { step size. An additional digital zoom was } \\
\text { applied, resulting in a lateral resolution of } 0.07 \mu m \text {. } \\
\text { Transgenic mouse line (Thy1-GFP) expressing GFP } \\
\text { under the thy1 promoter. }\end{array}$ & $\begin{array}{l}\text { Imaging: Images of } \\
\text { dendrites in different } \\
\text { brain regions were acquired } \\
\text { under 561 } \mathrm{nm} \text { fluorescent } \\
\text { illumination using a } \\
\text { confocal microscope }\left(63^{\prime}\right. \\
\text { objective, } 1.4 \mathrm{NA}) \text { at a pixel } \\
\text { resolution of } 1024 \times 1024 \\
\text { with a } 3.43 \text { zoom, resulting } \\
\text { in a } 0.07 \mu \mathrm{m} \text { pixel size [25]. }\end{array}$ & $\begin{array}{l}\text { Imaging: Images } \\
\text { of dendrites in } \\
\text { different brain } \\
\text { regions were } \\
\text { acquired under } \\
\text { 488nm fluores-cent } \\
\text { illumination } \\
\text { using a 2-photon } \\
\text { microscope } \\
\text { (40'objective) } \\
\text { with a } 2.0 \text { zoom. }\end{array}$ \\
\hline Procedure & See $[25,26]$ & See $[25,27]$ & See $[28-31]$ \\
\hline Aparatus & $\begin{array}{l}\text { Fluorescent confocal microscopy equipped with live } \\
\text { cell imaging setup (Zeiss LSM780) }\end{array}$ & $\begin{array}{l}\text { Fluorescent } \quad \text { confocal } \\
\text { microscopy (Zeiss LSM800) }\end{array}$ & $\begin{array}{l}\text { 2-photon } \\
\text { microscopy (Zeiss } \\
\text { LSM } 7 \text { MP Axio } \\
\text { Examiner.Z1) }\end{array}$ \\
\hline
\end{tabular}


Table 2. Morphological features (volume, length, head width, neck length) and types of dendritic spines for each spine shown in Figure 4(a).

\begin{tabular}{cccccc}
\hline $\begin{array}{c}\text { Spine } \\
\text { ID }\end{array}$ & $\begin{array}{c}\text { Volume } \\
\left.\text { (micron }^{3}\right)\end{array}$ & $\begin{array}{c}\text { Length } \\
\text { (micron) }\end{array}$ & $\begin{array}{c}\text { Head Width } \\
\text { (micron) }\end{array}$ & $\begin{array}{c}\text { Neck Length } \\
\text { (micron) }\end{array}$ & Type \\
\hline 1 & 0.198 & 0.759 & 0.427 & 0.069 & Filopodium \\
2 & 0.491 & 0.331 & 0.662 & 0 & Stubby \\
3 & 1.444 & 0.531 & 1.021 & 0 & Stubby \\
4 & 2.241 & 1.725 & 1.173 & 0.207 & Mushroom \\
5 & 1.418 & 1.311 & 1.145 & 0.207 & Mushroom \\
6 & 0.383 & 0.289 & 0.579 & 0 & Stubby \\
7 & 0.568 & 0.276 & 0.552 & 0 & Stubby \\
8 & 1.484 & 0.579 & 0.966 & 0 & Stubby \\
9 & 0.499 & 0.379 & 0.759 & 0 & Stubby \\
10 & 0.947 & 0.400 & 0.800 & 0 & Stubby \\
11 & 0.473 & 0.331 & 0.662 & 0 & Stubby \\
12 & 0.422 & 0.276 & 0.552 & 0 & Stubby \\
13 & 1.060 & 0.524 & 0.800 & 0 & Stubby \\
14 & 1.025 & 1.035 & 0.924 & 0.138 & Mushroom \\
15 & 1.607 & 0.579 & 1.131 & 0 & Stubby \\
16 & 0.425 & 0.358 & 0.552 & 0 & Stubby \\
17 & 0.304 & 0.289 & 0.524 & 0 & Stubby \\
\hline
\end{tabular}

Table 3. Morphological features (volume, length, head width, neck length) and types of dendritic spines for each spine shown in Figure 5

\begin{tabular}{cccccc}
\hline $\begin{array}{c}\text { Spine } \\
\text { ID }\end{array}$ & $\begin{array}{c}\text { Volume } \\
\left.\text { (micron }^{3}\right)\end{array}$ & $\begin{array}{c}\text { Length } \\
\text { (micron) })\end{array}$ & $\begin{array}{c}\text { Head Width } \\
\text { (micron) }\end{array}$ & $\begin{array}{c}\text { Neck Length } \\
\text { (micron) }\end{array}$ & Type \\
\hline 1 & 0.847 & 1.38 & 0.855 & 0.552 & Mushroom \\
2 & 0.364 & 1.104 & 0.524 & 0.276 & Filopodium \\
3 & 0.728 & 0.524 & 0.855 & 0 & Stubby \\
4 & 1.395 & 2.208 & 0.966 & 0.966 & Mushroom \\
5 & 0.322 & 0.420 & 0.703 & 0 & Stubby \\
6 & 1.138 & 0.841 & 1.035 & 0 & Stubby \\
7 & 0.309 & 0.469 & 0.621 & 0 & Stubby \\
8 & 0.447 & 0.407 & 0.69 & 0 & Stubby \\
9 & 0.950 & 0.579 & 1.076 & 0 & Stubby \\
10 & 0.983 & 0.503 & 0.938 & 0 & Stubby \\
11 & 1.569 & 1.518 & 1.021 & 0.207 & Mushroom \\
12 & 1.379 & 3.864 & 0.469 & 0.483 & Filopodium \\
13 & 0.386 & 0.351 & 0.703 & 0 & Stubby \\
\hline
\end{tabular}

\section{Materials and Methods}

3dSpAn comprises four main modules: Preprocessing and ROI selection (M1), Intensity thresholding and seed selection (M2), Multiscale segmentation (M3) and Quantitative morphological feature extraction (M4). Figure 1 describes an overall workflow of 3dSpAn, the different components of GUI and a snapshot of selected regions of interest (ROI), for a confocal microscopic image of in vitro neuronal culture is used. 3dSpAn software is implemented in C++ language and Qt development environment [20]. For 3D visualization of the segmented spines, we used the open source software ITK SNAP [19]

\subsection{Preprocessing and ROI selection (M1)}

The preprocessing step is used to eliminate image noise (mainly salt and pepper noise) and to equalize image resolution along all three axes (confocal images have lower axial resolution than lateral resolution). 3D median filter [22] is applied to eliminate image noise (the median filter kernel size and voxel dimensions can be selected by the user, details are given in $\left.3 d S p A n \_S u p p l e m e n t a r y\right)$. Bilinear 
Table 4. Morphological features (volume, length, head width, neck length) and types of dendritic spines for each spine shown in Figure 6.

\begin{tabular}{cccccc}
\hline $\begin{array}{c}\text { Spine } \\
\text { ID }\end{array}$ & $\begin{array}{c}\text { Volume } \\
\left.\text { (micron }^{3}\right)\end{array}$ & $\begin{array}{c}\text { Length } \\
\text { (micron) }\end{array}$ & $\begin{array}{c}\text { Head Width } \\
\text { (micron) }\end{array}$ & $\begin{array}{c}\text { Neck Length } \\
\text { (micron) }\end{array}$ & Type \\
\hline 1 & 1.120 & 1.242 & 0.966 & 0.069 & Mushroom \\
2 & 0.329 & 0.897 & 0.662 & 0.138 & Mushroom \\
3 & 0.483 & 1.035 & 0.648 & 0.138 & Mushroom \\
4 & 1.917 & 1.311 & 0.621 & 0.414 & Mushroom \\
5 & 0.732 & 0.345 & 0.662 & 0 & Stubby \\
6 & 0.519 & 0.407 & 0.814 & 0 & Stubby \\
7 & 0.375 & 0.379 & 0.662 & 0 & Stubby \\
8 & 0.645 & 0.448 & 0.621 & 0 & Stubby \\
9 & 0.385 & 0.345 & 0.662 & 0 & Stubby \\
10 & 0.506 & 1.242 & 0.69 & 0.621 & Mushroom \\
11 & 0.509 & 0.759 & 0.469 & 0.069 & Mushroom \\
12 & 1.406 & 0.345 & 0.69 & 0 & Stubby \\
13 & 0.370 & 0.096 & 0.193 & 0 & Stubby \\
14 & 0.318 & 0.165 & 0.331 & 0 & Stubby \\
15 & 0.289 & 0.282 & 0.552 & 0 & Stubby \\
16 & 0.490 & 0.193 & 0.386 & 0 & Stubby \\
17 & 0.122 & 0.193 & 0.331 & 0 & Stubby \\
\hline
\end{tabular}

interpolation [23] is applied along the axial direction for appropriate scaling, producing smooth interpolating results in a real time. The user selects a region of interest (ROI) and for segmentation and quantitative morphological analysis of individual dendritic spine. The benefits of working with a smaller ROI is that the intensity thresholds are better estimated, and adjustment of these thresholds is also easier for a smaller ROI. After working on the current ROI, the user can select another ROI from the image and perform further segmentation and analysis. It is possible to stop in between the analysis process and to resume it later from the saved profile.

\subsection{Intensity thresholding and seed selection (M2)}

In order to perform the segmentation of dendritic spines, first we segment dendrite and spines together from the background, and then we segment individual spines from the dendrite. Generally, spines are of low intensity and dendrites are of higher intensity but spine and dendrite share a common intensity range. Two intensity thresholds for spine and dendrite, $t h_{s}$ and $t h_{d}$ respectively are initially estimated for the selected ROI $\left(R_{i}\right)$ in the following way. Let $\mu$ be mean intensity of the ROI and $\delta$ be standard deviation. The thresholds $t h_{s}$ and $t h_{d}$ are calculated as

$$
t h_{s}=\mu+\delta
$$

$$
t h_{d}=\frac{t h_{s}+255}{2}
$$

If intensity at some point $P(x, y, z), I(P)<t h_{s}$ then it is classified as a pure background point and if $I(P) \geq t h_{d}$ then it is classified as a pure dendrite.The intensity range between $t h_{s}$ and $t h_{d}$ is the shared intensity space between spine and dendrite. A monotonically increasing fuzzy membership function is used here to calculate spine and dendrite membership $\left(\mu_{s}\right.$ and $\left.\mu_{d}\right)$ of each pixel,

$$
\mu_{s}(P)=\left\{\begin{array}{cc}
0, & \text { if }(P)<t h_{s}, \\
\frac{t h_{d}-I_{P}}{t h_{d}-t h_{s}} & \text { ifth } \\
0, & \text { if }(P) \geq t h_{d},
\end{array}\right.
$$


105

$$
\mu_{d}(P)=\left\{\begin{array}{cc}
0, & \text { if }(P) \leq t h_{s} \\
\frac{I(P)-t h_{s}}{t h_{d}-t h_{s}}, & \text { ifth } h_{s}<I(P) \leq t h_{d} \\
1 & \text { otherwise. }
\end{array}\right.
$$

It may happen a spine in $R_{i}$ are of high intensity $\left(>t h_{d}\right.$ ) or a spine is disconnected from dendrite because of low intensity $\left(<t h_{s}\right)$. In this case we have to modify the value of $t h_{s}$ and $t h_{d}$ to segment the spines, accordingly. For some ROIs, we may encounter spines with higher intensities, and almost equal to the intensity of dendrite. In this case we have to increase the value of $t h_{d}$. The intensity space between $t h_{s}$ and $t h_{d}$ can be visualized as color transition from red to green, changing from spine to dendrite. This color coded visualization helps the user to modify $t h_{s}$ and $t h_{d}$ manually. To select a dendritic spine $\left(S_{i}\right)$ for segmentation, the user needs to place a seed point on it. Multiple seed points can be placed for a single spine $\left(S_{i}\right)$. The pixels with intensities greater than $t h_{d}$ are considered as implicit dendritic seeds. Explicit dendritic seeds are required if $\mu_{d}$ values are too low for voxels belonging to dendrite region. In this case, a separator is placed to separate two touching spines. Figure 2 shows intensity thresholding and a seed selection module on the image presented in Figure 1.

\subsection{Multiscale segmentation (M3)}

The user-specified seed points and separators are considered as inputs for the MSO algorithm to segment the dendrites and the spines. MSO algorithm segments two conjoint objects, namely spines from dendrites in this case, coupled at unknown locations and at arbitrary scales in the shared intensity space (bounded by $t h_{s}$ and $t h_{d}$ ). With user-specified seed points and separators, the MSO algorithm separates the spines from the dendrites at a specific scale based on fuzzy distance transform (FDT) and fuzzy morphoconnectivity strength. After segmentation at the specific scale, the previous separation boundary is frozen using constrained morphological dilation, enabling segmentation at the next, finer scale. In this iterative approach of MSO, it takes several iterations to grow path-continuity of an object starting from its seed, often falling in large-scale regions, to a peripheral location with fine scale details, see Saha et. al [3] for theoretical and mathematical details of MSO algorithm. Figure 3 shows the GUI multiscale segmentation module.

\subsection{Quantitative morphological feature extraction (M4)}

After 3D segmentation, the feature extraction module extracts key morphological features for each of the segmented spine $S_{i}$, like volume, length, head width, and neck length. These features are extracted by identifying three characteristic points for spines, 1) the central base point, i.e., the central point of the junction between the segmented spine and dendrite, 2) the central head point, i.e., the locally deepest point in the spine, and 3) the farthest point on the spine from the central base point, determined using FDT based shortest path. Using these features, each $S_{i}$ is categorized into one of the three major spine classes: stubby, filopodia or mushroom spines. For the mathematical detail of the quantitative measurements and spine classification please refer to our previous paper [16]. Figure 4 shows the quantitative morphological feature extraction module.

\section{Conclusions}

The main advantage of 3dSpAn software is its ability to segment and reconstruct individual spine in 3D for images different modalities and significant artifact contents, obtained using different laboratory techniques. The software extracts quantitative morphological features for dendritic spines and classifies them into one of the three classes: stubby, filopodia and mushroom spines. Each spine shape reflects the function and the strength of synaptic connections, thus precise determination of spine morphology is crucial in studies of synaptic plasticity [18]. The segmentation results were shown in in vitro and ex vivo images captured using confocal microscopy and in vivo image captured using 
two photon microscopy. The high reproducibility of proposed segmentation method has been already established in our previous publications [1,24].

\section{Patents}

\section{Not Applicable}

Supplementary Materials: 3dSpAn source code, 3dSpAn executable (3dSpAn V1.2 Installer for Windows), Manual(3dSpAn_Supplementary.pdf), video tutorials, sample data are available online at: https://sites.google. $\mathrm{com} /$ view/3dSpAn/. The figures, tables etc. given in 3dSpAn_Supplementary.pdf are listed below.

Figure S1:The 3dSpAn GUI; Figure S2: A 3D image is loaded in the 3dSpAn software; Figure S3: Default Preprocessing option can be found in Tool menu of 3dSpAn GUI. Preprocessing settings dialog is shown in inset; Figure S4: Option to show Gridlines can be found in Display menu of the 3dSpAn GUI, Figure S5: Gridlines are displayed on the image loaded in 3dSpAn GUI; Figure S6: Options to select a region and crop that are enclosed with red boxes; Figure S7: Cropped region of the loaded 3D image is shown in the 3dSpAn GUI. The cropped region is named as ROI_1; Figure S8: The cropped ROI is magnified. The spin boxes enclosed by red box is used to tune the Pure Spine and Pure Dendrite intensity range; Figure S9: Option to show display the ROI as color-coded fuzzy segment can be found Display menu of the 3dSpAn GUI; Figure S10: The image is shown as color coded fuzzy segment depending on the pure spine and pure dendrite range; Figure S11: The image is shown as color coded fuzzy segment depending on the pure spine and pure dendrite range and the option to select seed type and add seed is enclosed by red boxes; Figure S12: A spine (encircled by red circle) is shown with pixel having intensity (119) greater than the shared intensity range upper bound (70) and falls in pure dendrite region; Figure S13: The upper bound of the shared intensity space between spine and dendrite is increased to 125.To see the added seeds, uncheck the "Fuzzy Segment" from Display->Fuzzy Segment; Figure S14: The option to add spine/dendrite/separator can be found from Display->Show Seed/Sep. Spine seeds are shown in red and dendrite seeds are shown in green; Figure S15: The option to Show Seed Id can be found from Display->Show Seed Id->Spine/Dendrite/Separator; Figure S16: To run the segmentation algorithm with user given seeds and separators user need to click on the Execute button, enclosed by red box; Figure S17: Segmented spines are shown in red and dendrite is shown in green; Figure S18: Click on Extract Feature (enclosed by red box) from the Analyse tab to calculated the morphological features of the segmented spine. After clicking Extract Feature segmented spine will be numbered in the order first spine seed marking; Figure S19: Check the option "Show Original Image" to go back to the full 3D image. Segmented spines are shown in red and segmented dendrite is shown in green; Figure S20: User can select a new ROI from the same image by checking the option Select a region and crop it by clicking on Crop button; Figure S21: The new ROI (ROI_2) is also segmented and numbered in the same way as ROI_1; Figure S22: The full image is shown with two segmented ROIs (ROI_1 and ROI_2). User can delete a ROI by putting ROI no. in the ROI No. and clicking Delete ROI button; Figure S23: After loading the image, if we load seeds/separators and previously cropped regions information from the option File->Load All ROI Profile, then the segmented regions will shown enclosed by blue boxes; Figure S24: The ITK Snap GUI. New image can be loaded from File->Open Main Image; Figure S25: The ITK Snap GUI. If we click File->Open Main Image, then the dialog box will appear; Figure S26: The ITK Snap GUI. After selecting the image, choose Raw Binary from the File Format drop down; Figure S27: After selecting the image, input proper Image dimensions, Voxel type and click Next> button; Figure S28: Click Finish to complete the image loading; Figure S29: The loaded 3D image is shown in three different plane; Figure S30: To load the same image as segmentation go to Segmentation->Open Segmentation; Figure S31: After loading the image as segmentation, spine and dendrite will be shown in different color (may not be always red and green). User can select the color for spine and dendrite intensity from color picker (enclosed by red circle). To see the 3D rendering click update button, enclosed by red box; Figure S32: All the three different plane views and 3D rendering window is shown together. To maximize 3D rendering window click on the 3D button enclosed by red box; Figure S33: 3D rendering of the segmented spine and dendrite. Table S1: Volume, Length, Head Width and type of the segmented spines ROI_2 shown in the Figure S21. Video S1: 3dSpAn Tuttorial-1; Video S2: 3dSpAn Tutorial-2; Video S3: 3D rendering of the segmented spine and dendrite.

Author Contributions: S.B. and J.W. conceived the study; S.B., P.K.S., D.P., J.W. performed the experimental design; N.D., S.B. developed the software; N.D., E.B., M.B., A.Z., B.R. analyzed the data; S.B., N.D., B.R, E.B., J.W, E.P.,M.B. wrote the manuscript.

Funding: This project was supported by the Department of Biotechnology, India grant: BT/PR16356/BID/7/596/2016 (S.B) and the Polish National Science Centre, grant 2017/26/E/NZ4/00637 (J.W.). N.D. acknowledges CSIR SRF Fellowship, File No. 09 I 096(0921)2K18 EMR-I), India. E.B. acknowledges Polish National Science Centre grant UMO-2017/27/N/NZ3/02417. M.B. acknowledges Foundation for Polish Science grant POIR.04.04.00-00-43BC/17-00. D.P. has been supported by Polish National Science Centre (2019/35/O/ST6/02484, 2014/15/B/ST6/05082), Foundation for Polish Science co-financed by the European Union under the European Regional Development Fund (TEAM to DP). The work was co-supported by European Commission Horizon 2020 Marie Skłodowska-Curie ITN Enhpathy grant 'Molecular Basis of Human enhanceropathies'; and National Institute of Health USA 4DNucleome grant 1U54DK107967-01 “Nucleome Positioning System for Spatiotemporal Genome Organization and Regulation. E.P. was supported by the Deutsche Forschungsgemeinschaft (DFG) grant PO732. 
bioRxiv preprint doi: https://doi.org/10.1101/864587; this version posted December 27, 2020. The copyright holder for this preprint (which was not certified by peer review) is the author/funder. All rights reserved. No reuse allowed without permission.

Acknowledgments: Authors acknowledge CMATER Laboratory, Department of Computer Science and Engineering, Jadavpur University, Kol-32 for supporting the development.

Conflicts of Interest: The authors declare that they have no conflict of interests.

\author{
Abbreviations \\ The following abbreviations are used in this manuscript: \\ MSO Multiscale Opening \\ FDT Fuzzy Distnace Transform \\ ROI Region of Interest
}

\title{
Appendix A
}

\section{References}

1. Basu, S; Saha, P. K.; Roszkowska, M; Magnowska, M; Baczynska, E; Das, N; Plewczynski, D; Wlodarczyk, J. Quantitative 3-D morphometric analysis of individual dendritic spines. Sci. Rep. 2018, 8.

2. Worbs, T; Foster, R. 4D tracking with Imaris. Oxford Instruments, Bitplane Imaris 2007, http:/ / www.bitplane. com/learning/4d-tracking-with-imaris-immunology.

3. Saha, P K; Basu, S; Hoffman, E A. Multiscale Opening of Conjoined Fuzzy Objects: Theory and Applications. IEEE Transactions on Fuzzy Systems 2016, 24, 1121-1133.

4. Harris, K M; Kater, S B. Dendritic Spines: Cellular Specializations Imparting Both Stability and Flexibility to Synaptic Function. Annual Review of Neuroscience 1994, 17, 341-371.

5. Lee, K F. H.; Cary, S; Béïque, J C. Examining form and function of dendritic spines. In Neural Plasticity, Hindawi Publishing Corporation, 2012.

6. Matsuzaki, M;Honkura, N; Ellis-Davies, G. C.R. and Kasai, H. Structural basis of long-term potentiation in single dendritic spines. Nature 2004, 429, 761-766.

7. Caroni, P; Donato, F; Muller, D. Structural plasticity upon learning: regulation and functions. Nature Reviews Neuroscience 2012, 13, 478-490.

8. Sala, C; Segal, M. Dendritic Spines: The Locus of Structural and Functional Plasticity. Physiological Reviews 2014, 94, 141-188.

9. Fiala, J C; Spacek, J; Harris, K M. Dendritic spine pathology: Cause or consequence of neurological disorders?. Brain Research Reviews 2002, 39, 29-54.

10. Chidambaram, S Babu; Rathipriya, A. G.; Bolla, S Rao; Bhat, A; Ray, B; Mahalakshmi, A Marappa; Manivasagam, T; Thenmozhi, A Justin; Essa, M Mohamed; Guillemin, G J.; Chandra, R; Sakharkar, M Kishore. Dendritic spines: Revisiting the physiological role. Progress in Neuro-Psychopharmacology and Biological Psychiatry 2019, 92, 161-193.

11. Spires, T L.;Meyer-Luehmann, M; Stern, E A.; McLean, P J.; Skoch, J; Nguyen, P T.;Bacskai, B J.;Hyman, B T. Dendritic spine abnormalities in amyloid precursor protein transgenic mice demonstrated by gene transfer and intravital multiphoton microscopy. Journal of Neuroscience 2005, 25, 7278-7287.

12. Glausier, J. R.; Lewis, D. A. Dendritic spine pathology in schizophrenia. Neuroscience 2013, 251, 90-107.

13. Wong S T. C.; Chen, X; Xu, X. Methods and systems for the analysis of $3 \mathrm{~d}$ microscopic neuron images. 2006, http:/ / www.google.com.gt/patents/WO2006125188A1?cl=en.

14. Ruszczycki, B; Wlodarczyk, J; Kaczmarek, L. Method and a system for processing an image comprising dendritic spines. 2012, https:/ / patents.google.com/patent/US20140169647.

15. Ruszczycki, B; Szepesi, Z; Wilczynski, G M; Bijata, M; Kalita, K;Kaczmarek, L; Wlodarczyk, J. Sampling issues in quantitative analysis of dendritic spines morphology. BMC Bioinformatics 2012, 13, 213.

16. Basu, S; Plewczynski, D; Saha, S; Roszkowska, M; Magnowska, M; Baczynska, E; Wlodarczyk, J. 2dSpAn: semiautomated 2-d segmentation, classification and analysis of hippocampal dendritic spine plasticity. Bioinformatics 2016, 32, 2490-2498.

17. Swanger, S A; Yao, X; Gross, C; Bassell, G J. Automated 4D analysis of dendritic spine morphology: applications to stimulus-induced spine remodeling and pharmacological rescue in a disease model. Molecular Brain 2011, 4, 38. 
18. Kasai, H; Matsuzaki, M; Noguchi, J; Yasumatsu, N; Nakahara, H. Structure-stability-function relationships of dendritic spines. Trends in Neurosciences 2003, 26, 360-368.

19. Yushkevich, P A; Piven, J; Hazlett, H C; Smith, R G; Ho, S; Gee, J C and Gerig, G. User-guided 3D active contour segmentation of anatomical structures: significantly improved efficiency and reliability. Neuroimage 2006, 31, 1053-8119.

20. Qt Cross-platform software development for embedded and desktop. https://www.qt.io/, Accessed On: 2019-11-05.

21. Comery, T A.; Stamoudis, C X.; Irwin, S A; Greenough, W T. Increased density of multiple-head dendritic spines on medium-sized spiny neurons of the striatum in rats reared in a complex environment. Neurobiology of Learning and Memory 1996, 66, 93-96.

22. Brownrigg, D. R.K.. The Weighted Median Filter. Communications of the ACM 1984, 27, 807-818.

23. Gribbon, K. T.; Bailey, D. G.A novel approach to real-time bilinear interpolation. In Proceedings, DELTA 2004 Second IEEE International Workshop on Electronic Design, Test and Applications, ,2004,126-131.

24. Krzystyniak, A; Baczynska, E; Magnowska, M; Antoniuk, S; Roszkowska, M; Zareba-Koziol, M; Das, N; Basu, S; Pikula, M; Wlodarczyk, J. Prophylactic Ketamine Treatment Promotes Resilience to Chronic Stress and Accelerates Recovery: Correlation with Changes in Synaptic Plasticity in the CA3 Subregion of the Hippocampus. International Journal of Molecular Sciences 2019, 20, 1726.

25. Magnowska, M; Gorkiewicz, T; Suska, A; Wawrzyniak, M; Rutkowska-Wlodarczyk, I; Kaczmarek, L; Wlodarczyk, J. Transient ECM protease activity promotes synaptic plasticity. Scientific reports 2016, 6, 27757.

26. Szepesi, Z; Bijata, M; Ruszczycki, B; Kaczmarek, L; Wlodarczyk, J. Matrix metalloproteinases regulate the formation of dendritic spine head protrusions during chemically induced long-term potentiation. PLoS One 2013, 8, 63314.

27. Kim, B G; Dai, H; McAtee, M; Vicini, S; Bregman, B S. Labeling of dendritic spines with the carbocyanine dye DiI for confocal microscopic imaging in lightly fixed cortical slices. Journal of neuroscience methods 2007, $162,237-243$.

28. Łukasiewicz, K; Robacha, M; Bożycki, Ł; Radwanska, K; Czajkowski, R. Simultaneous two-photon in vivo imaging of synaptic inputs and postsynaptic targets in the mouse retrosplenial cortex. Journal of Visualized Experiments 2016, 109, e53528.

29. Trachtenberg, J T; Chen, B E; Knott, G W; Feng, G; Sanes, J R; Welker, E; Svoboda, K. Long-term in vivo imaging of experience-dependent synaptic plasticity in adult cortex. Nature 2002, 420, 788.

30. Holtmaat, A; de Paola, V; Wilbrecht, L; Trachtenberg, J T; Svoboda, K; Portera-Cailliau, C. Imaging neocortical neurons through a chronic cranial window. Cold Spring Harbor Protocols 2012, 2012, pdb-prot069617.

31. Chow, D K; Groszer, M; Pribadi, M; Machniki, M; Carmichael, S T; Liu, Xin; Trachtenberg, J T. Laminar and compartmental regulation of dendritic growth in mature cortex. Nature neuroscience 2009, 12, 116.

32. Edril E, Argunsah A. O.; Tasdizen, T; Unay, D; Cetin, M. A joint classification and segmentation approach for dendritic spine segmentation in 2-photon microscopy images in IEEE 12th International Symposium on Biomedical Imaging (ISBI) 2015, 797-800.

33. Smirnov MS; Garrett TR; Yasuda R. An open-source tool for analysis and automatic identification of dendritic spines using machine learning. PLoS One 2018,13(7).

34. Xiao, X.; Djurisic, M.; Hoogi, A.; Sapp, R.W.; Shatz, C.J; Rubin, D.L. Automated dendritic spine detection using convolutional neural networks on maximum intensity projected microscopic volumes.Journal of neuroscience methods 2018, 309, 25-34.

35. Xiao, C.; Li, W.; Deng, H.; Chen, X.; Yang, Y.; Xie, Q; Han, H. Effective automated pipeline for 3D reconstruction of synapses based on deep learning. BMC bioinformatics2018,19(1), p.263.

36. Driscoll, M.K.; Welf, E.S.; Jamieson, A.R.; Dean, K.M.; Isogai, T.; Fiolka, R; Danuser, G. Robust and automated detection of subcellular morphological motifs in 3D microscopy images. Nature methods2019, pp.1-8.

\section{Sample Availability: NA}

(C) 2020 by the authors. Submitted to Journal Not Specified for possible open access publication under the terms and conditions of the Creative Commons Attribution (CC BY) license (http://creativecommons.org/licenses/by/4.0/). 